\title{
A SHORT TUTORIAL ON NETWORK CALCULUS II: MIN-PLUS SYSTEM THEORY APPLIED TO COMMUNICATION NETWORKS
}

\author{
Jean-Yves Le Boudec, Patrick Thiran and Silvia Giordano \\ ICA-DSC, EPFL \\ CH-1015 Lausanne, Switzerland \\ http: / / icawww.epfl.ch
}

\begin{abstract}
We model some queuing systems arising in guaranteed service networks (such as RSVP/IP or ATM) as non-linear minplus systems that can be bounded by linear systems. We apply this method to the window flow control problem previously studied by Chang, Agrawal and Rajan, to the optimal smoothing of video through a network offering guaranteed service. We revisit the greedy shaper, and we also show how the same method enables us to compute the losses in a shaper by modelling it as a linear min-plus system. Finally, we describe the time-varying shaper.
\end{abstract}

\section{INTRODUCTION}

This second of two companion papers reviews some results of network calculus at the level of system description [7, 8]. The notations we used are defined in [1]. The cornerstone of this paper is a central result of min-plus algebra which describes the solution of a system of inequations using the concept of closure of an operator [4] (Theorem 1 in Section 3). It is equivalent to the description of a system (or circuit) by a set of ordinary differential equations in traditional system theory.

We use this result to propose a systematic method for modeling a number of situations arising in communication networks. In Section 2 we present our framework for system modelling; it relies on standard concepts and results from min-plus algebra. Then we introduce a family of min-plus linear, time-varying and idempotent operators which are useful for modelling a number of systems. They can be used in particular to represent systems with losses.

We will apply this modelling and the results from Section 3 to the following examples.

\section{Example 1: Greedy lossless traffic shaper}

Let us return to the example of a greedy shaper. We have seen in [1] that a greedy shaper is a device that forces an input flow $a(t)$ to have an output $x(t)$ which has $\sigma$ as arrival curve, at the expense of possibly delaying bits in the buffer. We assume that that $\sigma$ is sub-additive and such that $\sigma(0)=0$
(If it is not the case, $\sigma$ should be replaced by its sub-additive closure [1] $\bar{\sigma}=\inf \left\{\delta_{0}, \sigma, \sigma \otimes \sigma, \ldots, \sigma^{(n)}, \ldots\right\}$ where $\sigma^{(n)}=\sigma \otimes \ldots \otimes \sigma(n$ times $)$ and $\delta_{0}$ is the 'impulse' function defined by $\delta_{0}(t)=\infty$ for $t>0$ and $\delta_{0}(0)=0$ ).

The output of the shaper is therefore such that

$$
\begin{aligned}
x(t) & \leq a(t) \\
x(t)-x(s) & \leq \sigma(t-s)
\end{aligned}
$$

for all $0 \leq s \leq t$. The shaper is greedy if $x$ is the maximal solution of this set of inequalities. We have seen in [1] that $x=\sigma \otimes a$. We will show how this results from Theorem 1 .

\section{Example 2: Window flow controller}

This example is found independently in [2] and [3]. A data flow $a(t)$ is fed via a window flow controller to a network offering a service curve of $\beta$. The window flow controller limits the amount of data admitted into the network in such a way that the total backlog is less than or equal to $K$, where $K$ (the window size) is a fixed number (Figure 1).

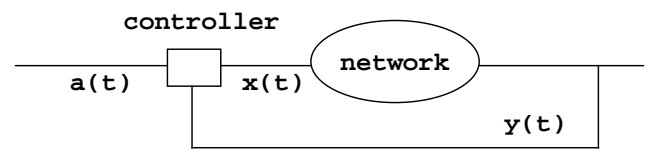

Figure 1: Example 2, from [2] or [3]

Call $x(t)$ the flow admitted to the network, and $y(t)$ the output. The definition of the controller means that $x(t)$ is the maximum solution to

$$
\left\{\begin{array}{l}
x(t) \leq a(t) \\
x(t) \leq y(t)+K
\end{array}\right.
$$

which implies that $x(t)=a(t) \wedge(y(t)+K)$. Note that we do not know the mapping $x(t) \rightarrow y(t)$, but we do know that

$$
y(t) \geq(\beta \otimes x)(t) .
$$

In [2], (1) and (2) are used to derive that

$$
x \geq \overline{(\beta+K)} \otimes a
$$


Equation (3) means that the complete system offers a service curve equal to the sub-additive closure $\overline{(\beta+K)}$. We show in this paper that this result is indeed obtained by min-plus methods.

\section{Example3: Optimal smoother}

We consider the transmission of variable bit rate (VBR) video over a network offering a guaranteed service such as ATM VBR or the guaranteed service of the IETF (see Figure 2). The guaranteed service requires that the flow produced by the output device conform with an arrival curve $\sigma$; in return, it receives a service guarantee expressed by a network service curve $\beta$. Functions $\sigma$ and $\beta$ are derived from the parameters used for setting up the reservation, for example, from the T-SPEC and R-SPEC fields used with the Resource Reservation Protocol (RSVP). In order to satisfy the smoothness (arrival curve) constraint constraint, the output of the encoder $a$ is fed to a smoother, possibly with some look-ahead. The resulting stream $x(t)$ is transported by the network; at the destination, the decoder waits for an initial playback delay $D$ and reads the stream $a(t-D)$ from the receive buffer.

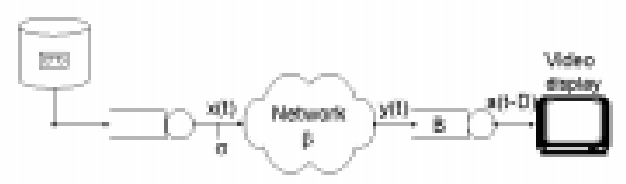

Figure 2: Example 3, optimal smoothing.

The smoother could be a greedy shaper: but this can be shown to be optimal only at the sender side. Here we consider another problem, namely, we would like to minimize the playback delay $D$ and the buffer size $B$ at the receiver. Another difference with shaping is that we allow our smoothing strategy to look-ahead, which a shaper does not.

Taking the convention that the time instant at which transmission begins is $t=0$ (so that $x(t)=0$ for $t \leq 0$ ), the constraints on the smoothed flow $x$ fed in the network are therefore that

$$
\begin{aligned}
& x(t) \leq \delta_{0}(t) \\
& x(t) \leq(x \otimes \sigma)(t)
\end{aligned}
$$

whereas the ones on the flow $y$ outputed from the network are that

$$
\begin{aligned}
& y(t) \geq a(t-D) \\
& y(t) \leq a(t-D)+B .
\end{aligned}
$$

Again, we do not know the exact mapping $x \rightarrow y$, but we know that the network offers a service curve $\beta$, so that $(x \otimes$ $\beta)(t) \leq y(t) \leq x(t)$. Using the deconvolution operator $\oslash$ and its properties, the set of constraints (6) and (7) can therefore be replaced by

$$
\begin{aligned}
& x(t) \geq(a \oslash \beta)(t-D) \\
& x(t) \leq a(t-D)+B .
\end{aligned}
$$

We will compute the smallest values of $D$ and $B$ that guarantee a lossless smoothing, and propose one smoothing strategy (which is not unique: another one is proposed in [5], achiveing the same optimal values of $D$ and $B$ ).

\section{Example 4: Losses in a shaper with finite buffer}

We reconsider Example 1, but now we suppose that the buffer is not large enough to avoid losses for all possible input traffic, and we would like to compute the amount of data lost at time $t$, with the convention that the system is empty at time $t=0$. We model losses as shown in Figure 3, where $x(t)$ is the data that has actually entered the system in the time interval $[0, t]$. The amount of data lost during the same period is therefore $L(t)=a(t)-x(t)$. The amount of data $(x(t)-x(s))$ that actually entered the system in any time interval $(s, t]$ is always bounded above by the total amount of data $(a(t)-a(s))$ that has arrived in the system during the same period. Therefore, for any $0 \leq s \leq t$, $x(t) \leq x(s)+a(t)-a(s)$ or equivalently

$$
x(t) \leq \inf _{u \text { such that } 0 \leq u \leq t}\{x(u)+a(t)-a(u)\} .
$$

On the other hand, $x$ is the part of $a$ that does actually enter the shaper, so the output of the shaper is $y=\sigma \otimes x$. There is no loss for $x(t)$ if $x(t)-y(t) \leq X$ for any $t$. Thus

$$
x(t) \leq y(t)+X=(\sigma \otimes x)(t)+X
$$

The data $x$ that actually enters the system is therefore the maximum solution to (10) and (11). In this paper we will provide an exact representation of $\mathrm{L}(\mathrm{t})$.

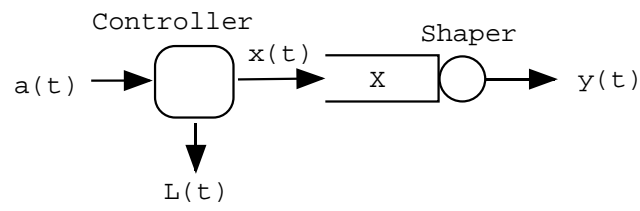

Figure 3: Example 4, shaper with losses

\section{Example 5: Time-varying shapers}

Given a function of two time variables $W(s, t)$, a time varying shaper forces the output $x(t)$ to satisfy the condition

$$
x(t) \leq x(s)+W(s, t)
$$

for all $s \leq t$, possibly at the expense of buffering some data. The shaper is optimal or greedy if it maximises its output among all possible shapers [7]. The time invariant greedy 
shaper of Example 1 is a special case that corresponds to $W(s, t)=\sigma(t-s)$.

We focus on the class of time varying shapers called time varying leaky bucket shapers, which are used for renegociating VBR connections between a user and a network operator. A time varying leaky bucket shaper is defined by a given number $J$ of leaky bucket specifications with bucket rate $r^{j}$ and bucket depth $b^{j}$, where $j=1, \ldots, J$. At specified time instants $t_{i}, i=0,1,2, .$. , the parameters of the leaky buckets are modified. A time varying leaky-bucket shaper is completely defined by the number $J$ of leaky buckets; the time instants $t_{i}$ at which the parameters changes; the buckets parameters $\left(r_{i}^{j}, b_{i}^{j}\right)$, for each $j$ and each interval $I_{i}=\left(t_{i}, t_{i+1}\right]$. Our class of time varying shapers fits in that general framework. It corresponds to

$$
W(s, t)=\min _{1 \leq j \leq J}\left\{\int_{s}^{t} r_{j}(u) d u+b_{j}(t)\right\}
$$

with $r_{j}(t)$ and $b_{j}(t)$ defined as the instantaneous bucket rate and depth at time $t$, namely $r_{j}(t)=r_{j}^{i}$ and $b_{j}(t)=b_{j}^{i}$ for the index $i$ such that $t_{i}<t \leq t_{i+1}$. We will provide the input-output characterisation of the time varying leaky bucket shapers.

\section{SYSTEM MODELLING}

The examples above involve particular types of operators $\Pi$ : $\mathcal{F} \rightarrow \mathcal{G}$, which are

- Min-plus convolution:

$$
\begin{aligned}
\mathcal{C}_{\sigma}(x)(t) & =(\sigma \otimes x)(t) \\
& =\inf _{0 \leq s \leq t}\{\sigma(t-s)+x(s)\},
\end{aligned}
$$

- Min-plus deconvolution:

$$
\begin{aligned}
\mathcal{D}_{\sigma}(x)(t) & =(x \oslash \sigma)(t) \\
& =\sup _{u \geq 0}\{x(t+u)-\sigma(u)\}
\end{aligned}
$$

- Idempotent operator:

$$
h_{\alpha}(x)(t)=\inf _{0 \leq s \leq t}\{\alpha(t)-\alpha(s)+x(s)\} .
$$

- General time-varying linear operator:

$$
\begin{aligned}
\mathcal{H}_{W}(x)(t) & =(x \cdot W)(t) \\
& =\inf _{0 \leq s \leq t}\{W(s, t)+x(s)\}
\end{aligned}
$$

Note that $\mathcal{C}_{\sigma}$ and $h_{\alpha}$ are tow particular cases of $\mathcal{H}_{W}$, with $W(s, t)=\sigma(t-s)$ and $W(s, t)=\alpha(t)-\alpha(s)$ respectively.

We also define a set of properties, which are direct applications of [4]:
- $\Pi$ is isotone if $x(t) \leq y(t)$ for all $t$ always implies $\Pi(x)(t) \leq \Pi(y)(t)$ for all $t$. One can check that all four operators $\mathcal{C}_{\sigma}, \mathcal{D}_{\sigma}, h_{\alpha}, \mathcal{H}_{W}$ are isotone.

- $\Pi$ is causal if for all $t, \Pi(x)(t)$ depends only on $x(s)$ for $0 \leq s \leq t . \mathcal{C}_{\sigma}, h_{\alpha}, \mathcal{H}_{W}$ are causal, but not $\mathcal{D}_{\sigma}$.

- $\Pi$ is upper-semi-continuous if for any decreasing sequence of trajectories $\left(x^{i}(t)\right)$ we have $\inf _{i} \Pi\left(x^{i}\right)=$ $\Pi\left(\inf _{i} x^{i}\right) . \mathcal{C}_{\sigma}, h_{\alpha}, \mathcal{H}_{W}$ are upper semi-continuous.

- $\Pi$ is time-invariant if $y(t)=\Pi(x)(t)$ for all $t$ and $x^{\prime}(t)=x(t+s)$ for some $s$ always implies that for all $t \Pi\left(x^{\prime}\right)(t)=y(t+s) \cdot \mathcal{C}_{\sigma}$ and $\mathcal{D}_{\sigma}$ are time-invariant, but not $h_{\alpha}$ nor $\mathcal{H}_{W}$.

- $\Pi$ is min-plus linear if it is upper-semi-continuous and $\Pi(x+K)=\Pi(x)+K$ for all constant $K . \mathcal{C}_{\sigma}$, $h_{\alpha}$ and $\mathcal{H}_{W}$ are min-plus linear, but not $\mathcal{D}_{\sigma}$.

We recast now the five examples using these operators.

Example 1: Optimal lossless traffic shaper. The solution of the optimal shaper is the solution to

$$
x \leq a \wedge \mathcal{C}_{\sigma}(x)
$$

Example 2: Window flow controller. Define $\Pi$ as the operator that maps $x(t)$ to $y(t)$. From Equation (1), we derive that $x(t)$ is the maximum solution to

$$
x \leq a \wedge(\Pi(x)+K)
$$

The operator $\Pi$ can be assumed to be isotone, causal and upper-semi-continuous, but not necessarily linear. However, we know that $\Pi \geq \mathcal{C}_{\beta}$. We will exploit this formulation in Section 3.

Example 3: Optimal smoother. Constraints (4) to (9) can be recast as

$$
\begin{aligned}
& x(t) \leq \delta_{0}(t) \wedge \mathcal{C}_{\sigma}(x)(t) \wedge\{a(t-D)+B\} \\
& x(t) \geq \mathcal{D}_{\beta}(a)(t-D)
\end{aligned}
$$

Example 4: Losses in a shaper with finite buffer. All operators are linear. We know that $x \leq a$. Combining this relation with (10) and (11), we derive that $x$ is the maximum solution to

$$
x \leq a \wedge h_{a}(x) \wedge(\sigma \otimes x+X) .
$$

Example 5: Time-varying shapers. One directly has

$$
x \leq a \wedge \mathcal{H}_{W}(x) .
$$

\section{SPACE METHOD}

In this paper we apply results from [4] to the problems formulated in the previous section. 
Theorem 1 ([4], Theorem 4.70, item 6) Let $\Pi$ be an operator $\mathcal{F} \rightarrow \mathcal{G}$, and assume it is isotone and upper-semicontinuous. For any fixed function $a \in \mathcal{F}$, the problem

$$
x \leq \Pi(x) \wedge a
$$

has one maximum solution, given by

$$
x=\bar{\Pi}(a)=\inf \left\{a, \Pi(a), \Pi[\Pi(a)], \ldots, \Pi^{(n)}(a), \ldots\right\} .
$$

The theorem is proven in [4], though with some amount of notation, using the fixed point method. It can also easily be proven directly as follows. Consider the sequence of decreasing sequences defined by $x^{0}=a$ and $x^{n+1}=$ $\Pi\left(x^{n}\right) \wedge x^{n}, n \in \mathbb{N}$. Then $x^{*}=\inf _{n \in \mathbb{N}} x^{n}$ is a solution of (19) because $\Pi$ is upper-semi-continuous. Conversely, if $x$ is a solution, then $x \leq x^{n}$ for all $n$ because $\Pi$ is isotone and thus $x \leq x^{*}$. We call the application of this theorem the space method, because it is based on an iteration on complete trajectories $x(t)$.

Let us first apply the theorem to Example 1. The maximal solution of (13) is

$$
x=\overline{\mathcal{C}}_{\sigma}(a)=\overline{(\sigma \otimes a)}=\bar{\sigma} \otimes a=\sigma \otimes a,
$$

the latter equality resulting from the sub-additivity of $\sigma$.

Let us next apply the theorem to Example 2. We know now that (14) has one maximum solution and that it is given by

$$
x=\overline{(\Pi+K)}(a) .
$$

Now from (2) we have $\Pi(x)+K \geq \beta \otimes x+K$. One easily shows that $x \geq \overline{(\beta+K)} \otimes a$ which is Equation (3).

The maximal solution of Example 3, which is

$$
\begin{aligned}
x(t) & =\overline{\mathcal{C}}_{\sigma}\left(\delta_{0}(t) \wedge\{a(t-D)+B\}\right) \\
& =\sigma(t) \wedge\{(\sigma \otimes a)(t-D)+B\}
\end{aligned}
$$

is guaranteed to exist if and only if it always verifies (16), which yields that

$$
\begin{aligned}
\sup _{t}\{(a \oslash \beta)(t-D)-\sigma(t)\} & \leq 0 \\
\sup _{t}\{(a \oslash \beta)(t-D)-(\sigma \otimes a)(t-D)\} & \leq B .
\end{aligned}
$$

Working out these two inequalities, we get that the smallest playback delay and buffer sizes are

$$
\begin{aligned}
& D=\inf \{s \geq 0:(a \oslash(\beta \otimes \sigma))(-s) \leq 0\} \\
& B=((a \oslash a) \oslash(\beta \otimes \sigma))(0),
\end{aligned}
$$

which are achived by an optimal smoothing strategy implemented at thes ender side, being given by (20).

From Theorem 1, the solution of Example 4 is

$$
x=\overline{h_{a} \wedge \mathcal{C}_{\sigma_{X}}}(a)=\ldots=\overline{h_{a}\left[\mathcal{C}_{\sigma_{X}}\right]}(a)
$$

The amount of lost data in the interval $[0, t]$ is

$$
\begin{aligned}
L(t) & =a(t)-x(t)=\sup _{k \geq 0}\left\{\begin{array}{l}
\sup _{0 \leq s_{2 k} \leq \ldots \leq s_{2} \leq s_{1} \leq t} \\
\end{array}=\left\{\sum_{i=1}^{k}\left[a\left(s_{2 i-1}\right)-a\left(s_{2 i}\right)-\sigma\left(s_{2 i-1}-s_{2 i}\right)\right]\right\}-k X\right\} .
\end{aligned}
$$

Finally, the maximal solution of (18) in Example 5. is

$$
x=\overline{\mathcal{H}}_{W}(a)=a \cdot \bar{W}=\inf \{a, a \cdot W, a \cdot W \cdot W, \ldots\}
$$

For the time varying leaky bucket shaper (12), one can compute this function for $t \in I_{i}=\left(t_{i}, t_{i+1}\right]$ from the initial conditions $q^{j}\left(t_{i}\right)$ and $w\left(t_{i}\right)$, which are respectively the bucket level and the backlog that are found by the traffic arriving in the interval $I_{i}$ [6]. Denoting

$$
\sigma_{i}^{0}(u)=\min _{1 \leq j \leq J}\left[r_{i}^{j} u+b_{i}^{j}-q^{j}\left(t_{i}\right)\right]
$$

we have that for $t \in I_{i}=\left(t_{i}, t_{i+1}\right]$

$x(t)=\left\{\sigma_{i}^{0}\left(t-t_{i}\right)+x\left(t_{i}\right)\right\} \wedge\left\{\inf _{t_{i}<s \leq t}\left\{\sigma_{i}(t-s)+R(s)\right\}\right.$.

\section{REFERENCES}

[1] J. Y. Le Boudec, P. Thiran, 'A short tutorial on Network Calculus I: fundamental bounds in Communication Networks', Proc. ISCAS'2000, Geneva, May 2000.

[2] C.S. Chang. 'A filtering theory for deterministic traffic regulation', in Proc. Infocom'97, Kobe, Japan, April 1997.

[3] R. Agrawal and R. Rajan. 'Performance bounds for guaranteed and adaptive services', Technical report RC 20649, IBM, December 1996.

[4] F. Baccelli, G. Cohen, G. J. Olsder and J.-P. Quadrat. Synchronization and Linearity, An Algebra for Discrete Event Systems, Wiley, 1992.

[5] J. Y. Le Boudec, O. Verscheure 'Optimal Smoothing for Guaranteed Service' Technical report No. SSC/1998/32, SSC/EPFL, October 1998.

[6] S. Giordano and J. Y. Le Boudec 'On a Class of Time Varying Shapers with Application to the RVBR Service' Technical report, SSC/EPFL, Nov. 1998.

[7] C.S. Chang, R. L. Cruz, J. Y. Le Boudec, P. Thiran 'A Min-Plus System Theory for Constrained Traffic Regulation and Dynamic Service Guarantees' Technical report No. SSC/1999/027, Oct. 1999.

[8] J. Y. Le Boudec, P. Thiran 'Network Calculus viewed as a Min-plus System Theory applied to Communication Networks' Technical report No.98/276, DI/EPFL, 1998. 